\title{
Infrapatellar fat pad resection or preservation during total knee arthroplasty: a meta-analysis of randomized controlled trials
}

Changjiao Sun ${ }^{1}$, Xiaofei Zhang ${ }^{2}$, Woo Guan Lee ${ }^{3}$, Yan Tu${ }^{1}$, Huimin $\mathrm{Li}^{1}, \mathrm{Xu} \mathrm{Cai}^{i^{*+}}$ and Huadong Yang ${ }^{{ }^{* *}+}$

\begin{abstract}
Background: The infrapatellar fat pad (IPFP) or Hoffa's fat pad is often resected during total knee arthroplasty in order to improve visibility. However, the management of the IPFP during total knee arthroplasty (TKA) is the subject of an ongoing debate that has no clear consensus. The purpose of this review was to appraise if resection of the IPFP affects clinical outcomes.

Methods: We conducted a meta-analysis to identify relevant randomized controlled trials involving infrapatellar fat pad resection and infrapatellar fat pad preservation during total knee arthroplasty in electronic databases, including Web of Science, Embase, PubMed, Cochrane Controlled Trials Register, Cochrane Library, Highwire, CBM, CNKI, VIP, and Wanfang database, up to March 2020.

Results: Nine randomized controlled trials, involving 783 TKAs (722 patients), were included in the systematic review. Outcome measures included patellar tendon length (PTL), Insall-Salvati ratio (ISR), rate of anterior knee pain, Knee Society Scores (KSS), and knee range of motion. The meta-analysis identified a trend toward the shortening of the patellar tendon with IPFP resection at 6 months $(P=0.0001)$ and 1 year $(P=0.001)$. We found no statistical difference in ISR $(P=0.87)$, rate of anterior knee pain within 6 months $(p=0.45)$ and 1 year $(p=0.38)$, KSS at 1 year $(p=0.77)$, and knee range of motion within 6 months $(p=0.61)$ and 1 year $(0.46)$.

Conclusion: Based on the available level I evidence, we were unable to conclude that one surgical technique of IPFP can definitively be considered superior over the other. More adequately powered and better-designed randomized controlled trial (RCT) studies with long-term follow-up are required to produce evidence-based guidelines regarding IPFP resection.
\end{abstract}

Keywords: Total knee arthroplasty, Infrapatellar fat pad, Hoffa's fat pad, patellar tendon length, Insall-Salvati ratio

\footnotetext{
*Correspondence: suncjdoctor@163.com; yanghuadong2020@sina.com

${ }^{+} \mathrm{Xu}$ Cai and Huadong Yang contributed equally to this work and should be considered as co- Corresponding author.

'Department of Orthopedic, Beijing Tsinghua Changgung Hospital, School of

Clinical Medicine, Tsinghua University, No.168 Litang Road, Dongxiaokou

Town, Changping District, Beijing 102218, China

Full list of author information is available at the end of the article
}

C C The Author(s). 2020 Open Access This article is licensed under a Creative Commons Attribution 4.0 International License, which permits use, sharing, adaptation, distribution and reproduction in any medium or format, as long as you give appropriate credit to the original author(s) and the source, provide a link to the Creative Commons licence, and indicate if changes were made. The images or other third party material in this article are included in the article's Creative Commons licence, unless indicated otherwise in a credit line to the material. If material is not included in the article's Creative Commons licence and your intended use is not permitted by statutory regulation or exceeds the permitted use, you will need to obtain permission directly from the copyright holder. To view a copy of this licence, visit http://creativecommons.org/licenses/by/4.0/ The Creative Commons Public Domain Dedication waiver (http://creativecommons.org/publicdomain/zero/1.0/) applies to the data made available in this article, unless otherwise stated in a credit line to the data. 


\section{Introduction}

The infrapatellar fat pad (IPFP), also known as Hoffa's fat pad, is located in the anterior compartment of the knee between the joint capsule and synovium. Often, it is resected during total knee arthroplasty procedures to allow for better visualization during the surgeon's approach [1]. Resection of the IPFP is estimated to occur in around $88 \%$ of total knee arthroplasties (TKAs) [2]. However, the consequences of IPFP resection are the subject of an ongoing debate that has no clear consensus. There is mixed evidence regarding the risk of avascular necrosis following resection, with some studies suggesting compromising the blood supply to the patella leads to shortening of the patella tendon resulting in patella fracture $[3,4]$. Moreover, others indicate it does not negatively affect the blood supply $[5,6]$. Some studies indicate that removal of the fat pad could lead to increased post-operative stiffness $[7,8]$, while others suggest that there are no functional differences when comparing resection to preservation $[9,10]$. The IPFP is thought to play a role in the inflammatory process and contains nociceptive fibers and is a possible source of anterior knee pain [11, 12]. Therefore, preservation of the IPFP may explain an increased incidence of anterior knee pain beyond 6 months post-operatively [13]. Meanwhile, Ioan-Facsinay and Kloppenburg [14] found that increased pain in patients with IPFP resection, remaining damaged nerve fibers may also be the source of ongoing pain in the IPFP resection group.

Given knowledge about how different management of the IPFP affects patient outcomes is variable, further information regarding how to handle the IPFP during TKA is necessary. Thus, this meta-analysis aims to objectively evaluate the influence of IPFP resection and preservation on TKA patient outcomes; we conducted a meta-analysis of current published evidence.

\section{Methods}

The current meta-analysis was registered on PROSPERO (International Prospective Register of Systematic Reviews) and the registration number was CRD42020168616. The Cochrane Handbook for Systematic Reviews of Interventions PRISMA (Preferred Reporting Items for Systematic Reviews and Metaanalyses) guidelines were applied to assess the quality of the results published in all included studies to make sure the results of our meta-analysis reliable and veritable.

\section{Search strategy}

We conducted a meta-analysis to identify relevant randomized controlled trials involving IPFP resection and preservation technique in total knee arthroplasty in electronic databases, including Web of Science, Embase, PubMed, Cochrane Controlled Trials Register, Cochrane Library, Highwire, CBM, CNKI, VIP, and Wanfang database, up to March 2020. The keywords used were "total knee arthroplasty," "total knee replacement," "infrapatellar fat pad (IPFP) or Hoffa's fat pad," "Hoffa's fat pad," "IPFP" in conjunction with Boolean operators "AND" or "OR." Review Manager Software was used to perform the meta-analysis.

\section{Inclusion criteria}

We included the study if it met the following inclusion criteria: (1) the intervention was the IPFP resection technique in TKA; (2) the comparator was the IPFP preservation technique; (3) the study designs were randomized controlled trial studies; (4) the outcomes were patellar tendon length (PTL), InsallSalvati ratio (ISR), rate of anterior knee pain (AKP), Knee Society Scores (KSS), and knee range of motion (ROM); (5) the included studies were required to contain at least one outcome; and (6) the studies must have had a follow-up rate of at least $80 \%$.

The exclusion criteria were as follows: (1) observational studies, (2) non-RCTs, and (3) studies with insufficient outcome data.

\section{Data extraction process}

Two authors independently extracted the available data from each study. Disagreements were resolved by discussion to reach consensus. We extracted the primary data based on the following: first author, year of publication, number of TKAs and participants, age, gender, primary indication for TKA, follow-up time, primary outcome, prothesis, and patellar resurfacing. The primary outcome consisted of PTL, ISR, rate of AKP, and KSS. Secondary outcomes included knee ROM.

\section{Assessment of studies}

According to the Cochrane Handbook for Systematic Reviews of Interventions, the methodological quality and basis of the included studies were assessed as follows: randomization, allocation concealment, blind method, selective reporting, group similarity at baseline, incomplete outcome data, compliance, timing of outcome assessments, and intention-to-treat analysis.

\section{Statistical analysis}

We use Review Manager Software for MAC (version 5.3) to perform the meta-analysis. The chi-square was used to assess the significance of heterogeneity. $I^{2}$ value $>50 \%$ suggested a high degree of heterogeneity; thus, we used the randomized-effects model. 
Otherwise, we used the fixed-effects model. The mean difference (MD) or standard MD was used to assess continuous outcomes such as PTL, ISR, KSS, and knee ROM with a 95\% confidence interval (CI). Relative risks with a $95 \% \mathrm{CI}$ were used to assess dichotomous outcomes such as the rate of AKP. If $P$ values were less than 0.05 , we considered the results as a statistically significant difference.

\section{Results}

\section{Search results}

The literature search and selection process are shown in Fig. 1. The literature search identified 248 citations. Of these, 112 duplicates were removed. Upon review of titles and abstracts of the 136 remaining articles, we excluded 123 papers according to the inclusion criteria; the full text of 13 articles was retrieved. Because sufficient data were not available in one article, three studies were non-RCTs; hence, four studies were excluded. Finally, we identified 783 TKAs (722 patients) assessed in 9 randomized controlled trials [1, 9, 15-20]. We presented the detailed baseline characteristics and general intervention information in Table 1. All the articles were published in English and Chinese between the years 2003 and March 2020.

\section{Risk of bias assessment}

The risk of bias summary and risk of bias graph for RCTs are shown in Figs. 2 and 3, respectively. Six studies adequately described the correct randomization and sufficient allocation concealment. Nine studies described the blinding of outcome assessment, and three studies described the blinding of participants and personnel. All studies retained complete outcome data and avoided selective reporting. We cannot ignore other potential risks of biases of all studies. Therefore, we rated as having an unclear risk of other bias. As a result, the

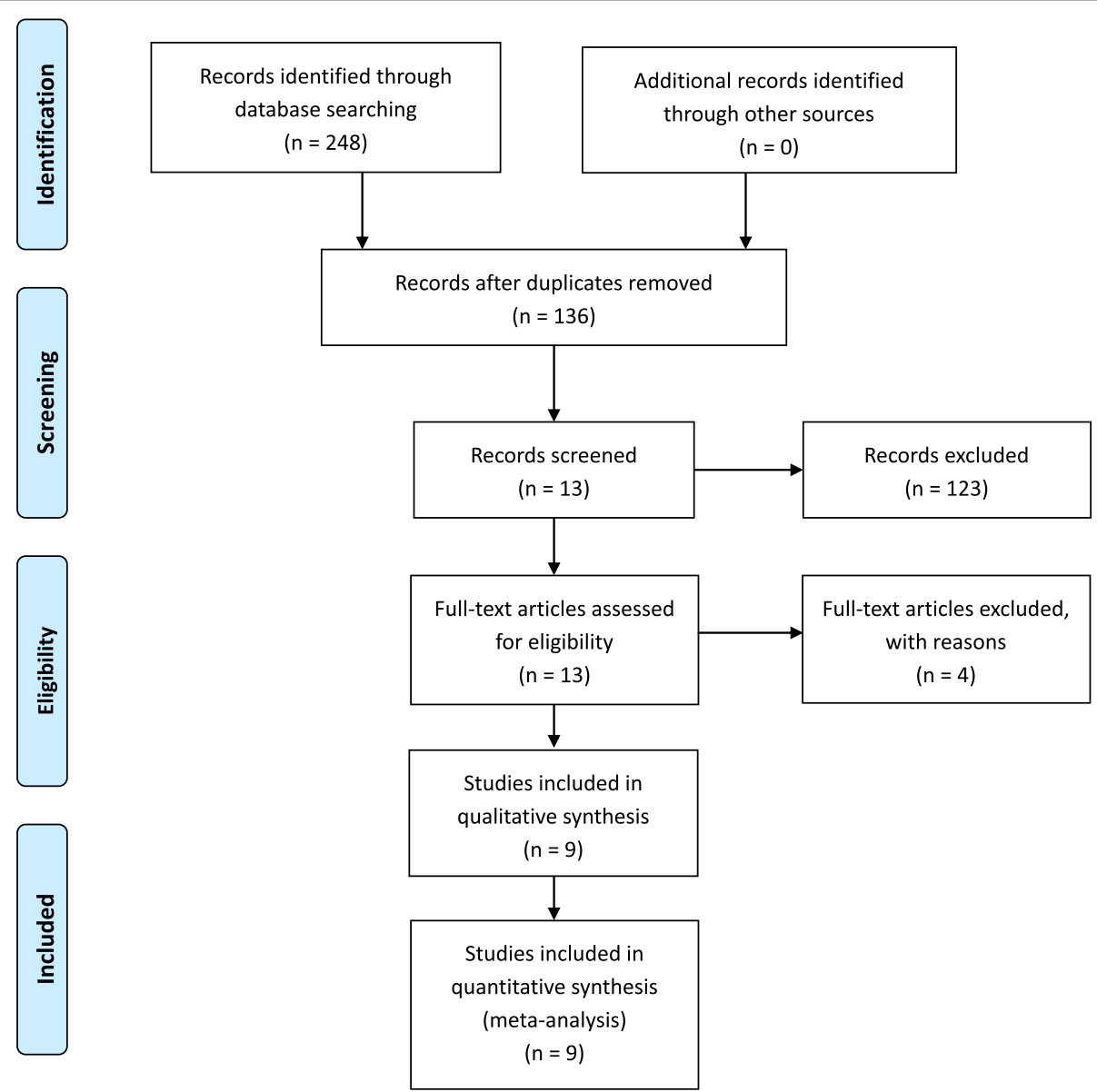

Fig. 1 The search results and selection procedure. Legend: The literature search identified 248 citations. Of these, 112 duplicates were removed. Upon review of titles and abstracts of the 136 remaining articles, we excluded 123 papers according to the inclusion criteria; the full text of 13 articles was retrieved. Because sufficient data were not available in one article, three studies were non-RCTs; hence, four studies were excluded. Finally, we identified 783 TKAs (722 patients) assessed in 9 randomized controlled trials 


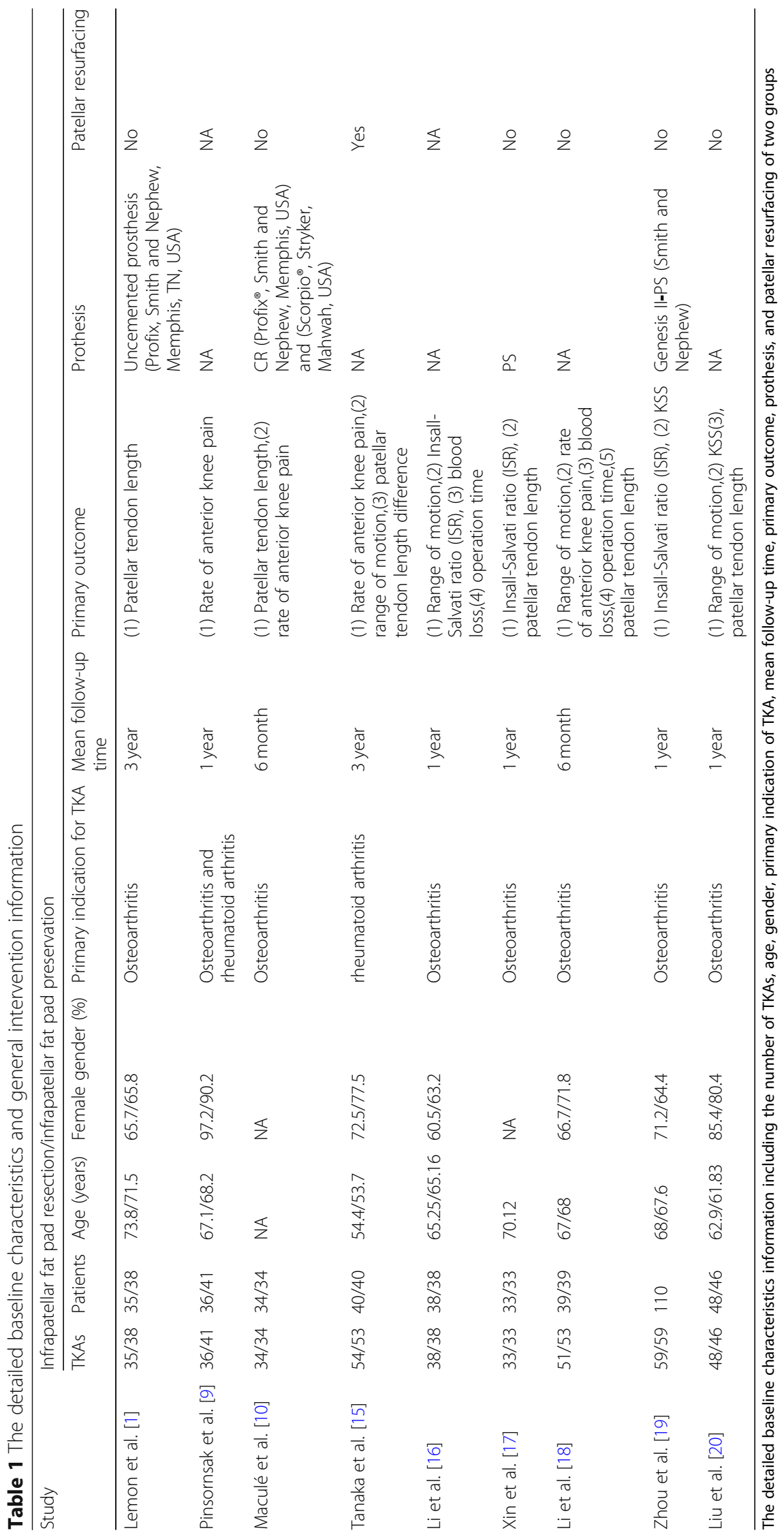




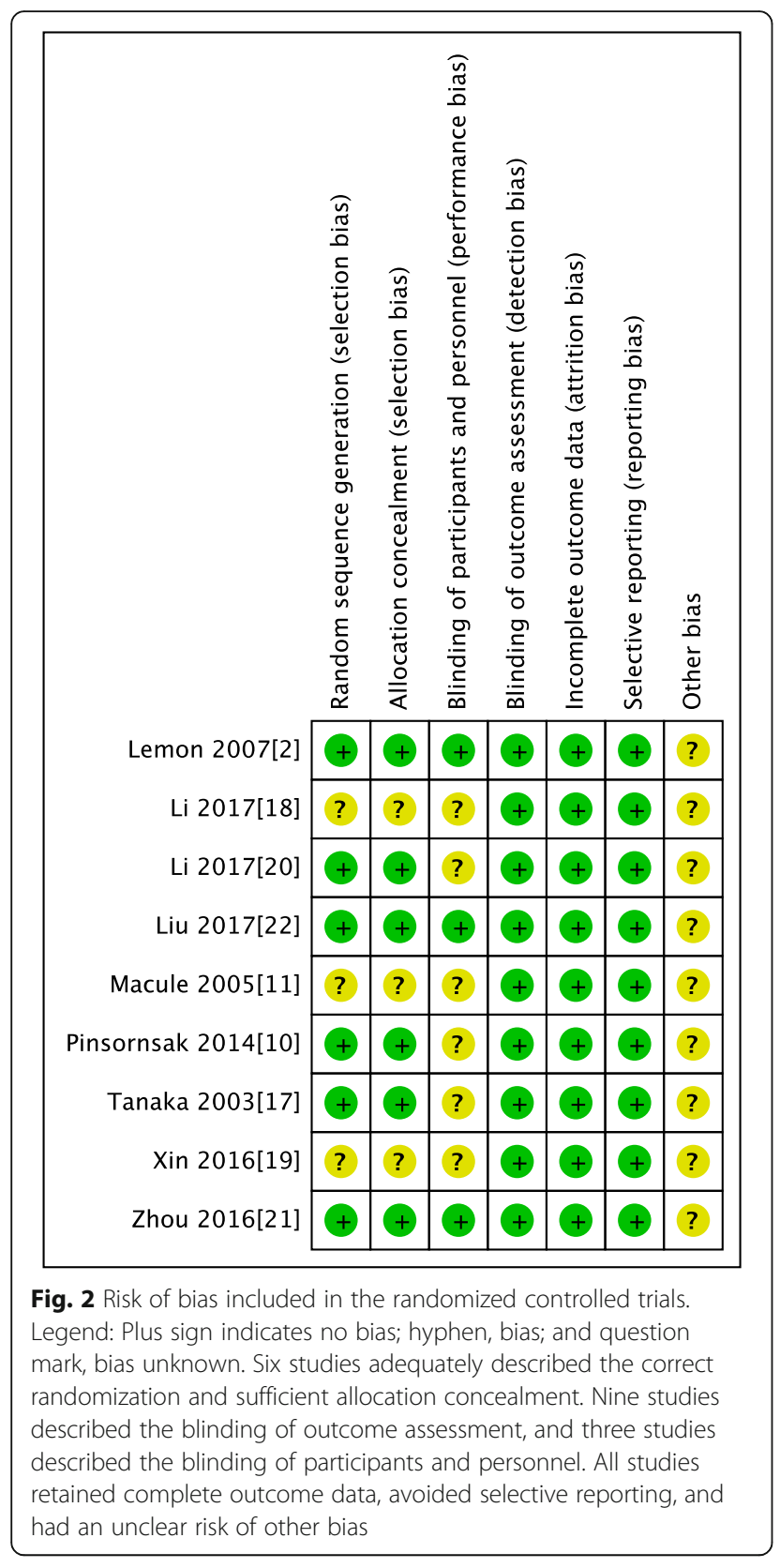

overall quality of the included studies was considered adequate (Figs. 2 and 3).

\section{Pooled analysis of PTL between IPFP resection and IPFP preservation}

Four RCTs reported the PTL at 6 months, and three RCTs reported PTL at 1 year. The pooled results showed that a trend toward shortening of the patellar tendon with IPFP resection at 6 months (MD $0.9,95 \%$ CI $[-1.36,-0.45], P=0.0001$; Fig. 4) and 1 year $(\mathrm{MD}-2.68,95 \%$ CI $[-4.32,-1.04], \mathrm{P}=$ 0.001; Fig. 4). This finding was reported to be statistically significant.
Pooled analysis of ISR between IPFP resection and IPFP preservation

Three studies reported the ISR; we found some statistical heterogeneity between the two groups of ISR $\left(x^{2}\right.$ $=30.33 ; \mathrm{df}=2, P<0.00001 ; I^{2}=93 \%$; Fig. 5); thus, a random-effects model was used. The pooled results showed that patients in both groups experienced similar ISR $(\mathrm{MD}=-0.03,95 \% \mathrm{CI}[-0.12,0.08], P=0.87$; Fig. 5).

Pooled analysis of the rate of anterior knee pain between IPFP resection and IPFP

Three RCTs reported the rate of anterior knee pain within 6 months, and two RCTs reported the rate of anterior knee pain at 1 year. The pooled results showed that patients in both groups experienced similar rates of anterior knee pain within 6 months (MD 2.12, 95\% CI $[0.3,15.19], P=0.45$; Fig. 6 ) and 1 year (MD 0.59, 95\% CI $[0.18,1.94], P=0.38$; Fig. 6)

\section{Pooled analysis of KSS between IPFP resection and IPFP preservation}

Two studies reported KSS at 1 year; we found some statistical heterogeneity between the two groups $\left(x^{2}=4.49\right.$; $\mathrm{df}=1, P=0.04 ; I^{2}=77 \%$; Fig. 7 ), and thus a randomeffects model was used. The pooled results showed that patients in both groups experienced similar KSS (MD = $-0.48,95 \%$ CI [- 3.76, 2.80], $\mathrm{P}=0.77$; Fig. 7).

\section{Pooled analysis of knee range of movements between IPFP resection and IPFP preservation}

Two studies reported a knee range of movements within 6 months, and two studies reported a knee range of movements in 1 year. The pooled results showed that patients in both groups experienced a similar knee range of movements within 6 months $(\mathrm{MD}=-0.81,95 \% \mathrm{CI}$ $[-3.96,2.34], P=0.61$; Fig. 8$)$ and at 1 year $(\mathrm{MD}=1.16$, 95\% CI [- 1.91, 4.24], $P=0.46$; Fig. 8).

\section{Discussion}

This meta-analysis included nine RCTs that assessed 783 TKAs (722 patients) and directly compared the clinical effectiveness of IPFP resection and IPFP preservation. The pooled data indicated no difference between the two operation modes in terms of ISR, Rate of AKP within 6 months and at 1 year, KSS at 1 year and knee ROM within 6 months and at 1 year. But there is a trend toward shortening of the patellar tendon with IPFP resection at 6 months and 1 year. There are currently no formal national guidelines about IPFP resection or preservation during TKA. To our knowledge, this is the first meta-analysis of only RCTs comparing IPFP resection and IPFP preservation in primary TKA. We only found one meta- 


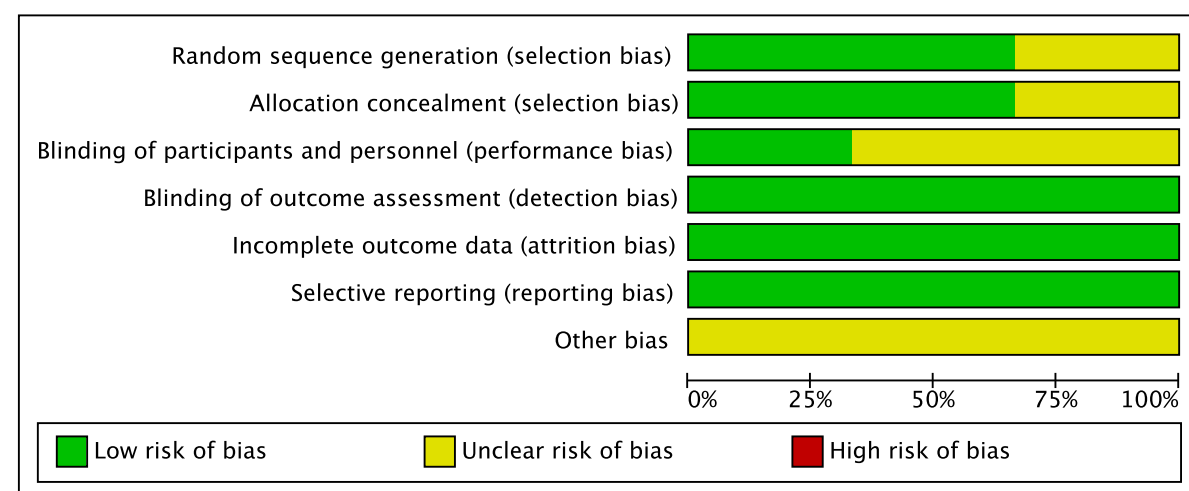

Fig. 3 The risk of bias graph. Legend: The overall quality of the included studies was considered adequate

analysis [21] comparing IPFP resection and IPFP preservation in primary TKA. However, the inclusion criteria of the previous meta-analyses included only 2 RCTs and five retrospective cohort studies design, which are potentially subject to selection bias.

Furthermore, the restriction of the previous metaanalyses to English language publications potentially limits the power that could be obtained with the inclusion of patient enrollment from non-English language studies. Finally, they did not analyze the ISR and knee range of motion. Thus, based on the current studies comparing IPFP resection and IPFP preservation in TKA, we only included level I trials (RCTs) in our metaanalysis, which may have a more convincing persuasive result. We include not only English studies but also Chinese RCTs. We have different results in terms of pain between our meta-analysis and previous metaanalysis. Moreover, we included ISR and knee range of motion, which would provide a more exact conclusion and could be a supplement for the previous metaanalysis.

The IPFP has been shown to contain peptidergic Cand substance $\mathrm{P}$ positive nerve fibers, which is thought to play a role in the inflammatory process and therefore is a possible source of anterior knee pain [11-13]. Meanwhile, the remaining damaged nerve fibers were also the source of ongoing pain in the IPFP Resection group. The meta-analysis by Nisar et al. [21] demonstrated a trend toward increased pain in the early 1 - to 2 -month post-operative period in the IPFP-P group. At 3 to 6 months, this trend is reversed with a higher incidence of knee pain in the

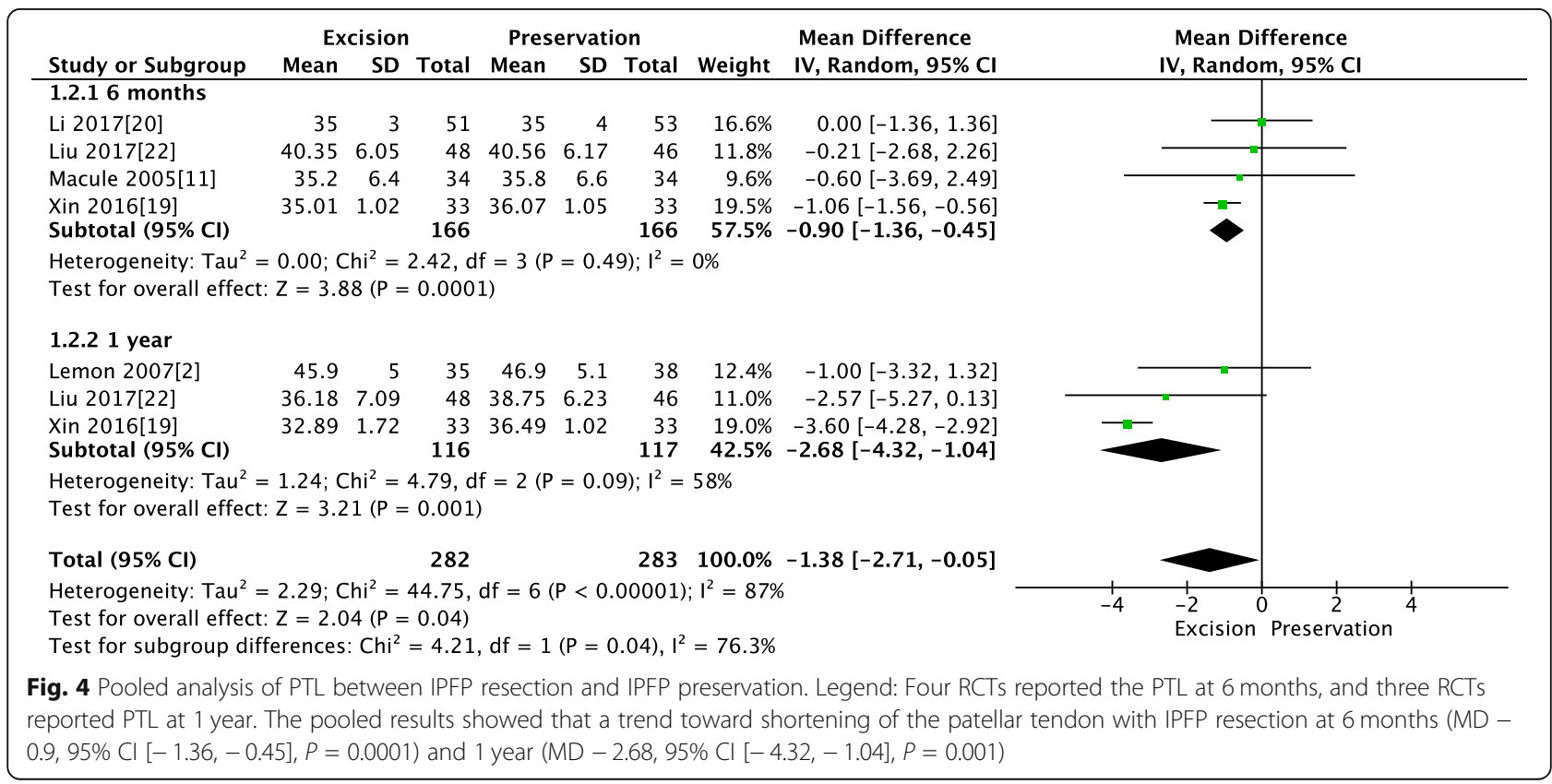




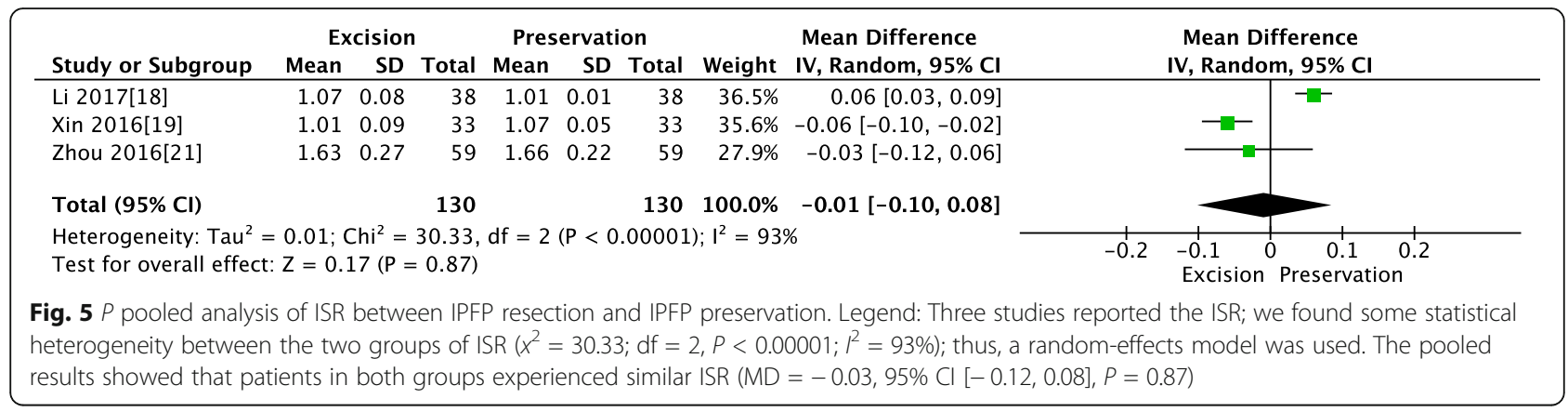

IPFP-R group. However, this trend is not supported by the present meta-analysis. In our meta-analysis, there is no statistical difference in the rate of anterior knee pain within 6 months and at 1 year. We think the change is likely due to seven recent RCTs providing new outcome data.

Our study showed a trend toward patellar tendon shortening in the IPFP resection group, which agreed with Nisar et al.'s result. When the IPFP is damaged, it undergoes fibrosclerotic change. Fibrous tissue bands pass through this area and create a non-extensile, rigid structure [22]. This, therefore, leads to the shortening of the patellar tendon. Some studies have shown that shortening of the patella tendon may result in a patella fracture $[3,4]$. However, no patella fracture occurred in all our IPFP resection groups.

ISR was one of the new findings of our metaanalysis compared to past meta-analyses. Our study has shown that ISR was not significantly changed following IPFP excision, which seems to contradict findings indicating that PTL was significantly shortened by resection in our study. This may be explained by the fact that the ISR index may not be a reliable parameter for post-operative TKA because osteophyte removal around the patellar bone affects the patellar bone length. However, other parameters (Blackburne-Peel ratio [23] and Caton-Deschamps [24]) are even less reliable for such measurements post-operatively because the joint line may change after TKA, which affects these parameters that required a consistent joint line for accurate evaluation [25].

Knee range of motion was another new finding of our meta-analysis compared to past meta-analyses. Tanaka et al.'s study reported a statistical decrease in flexion for the resection cohort [15]. However, there was evidence in our study suggesting that resection of the IPFP did not significantly affect knee flexion.

The utilization of the KSS was reported for the evaluation of post-operative function in three

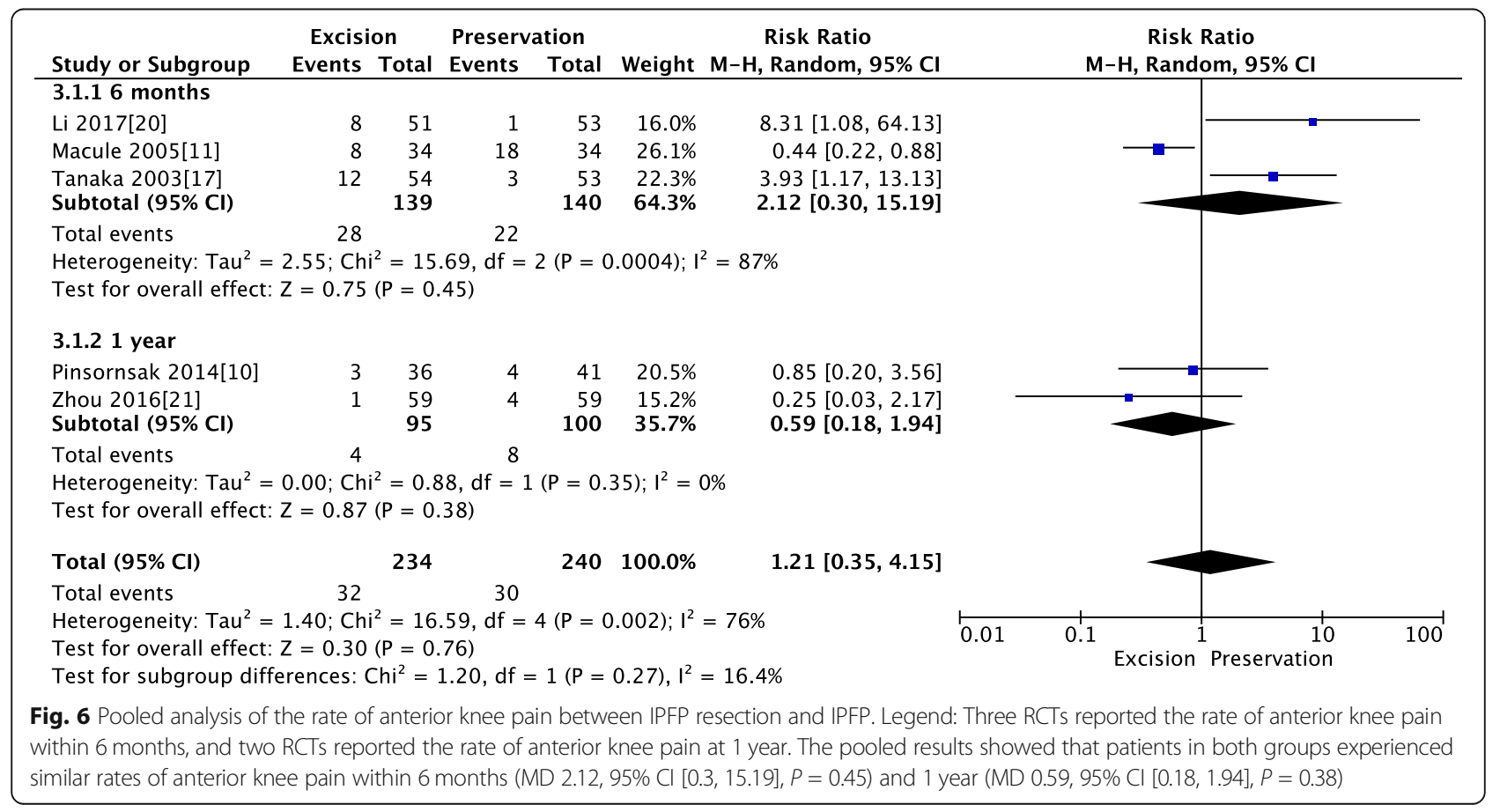




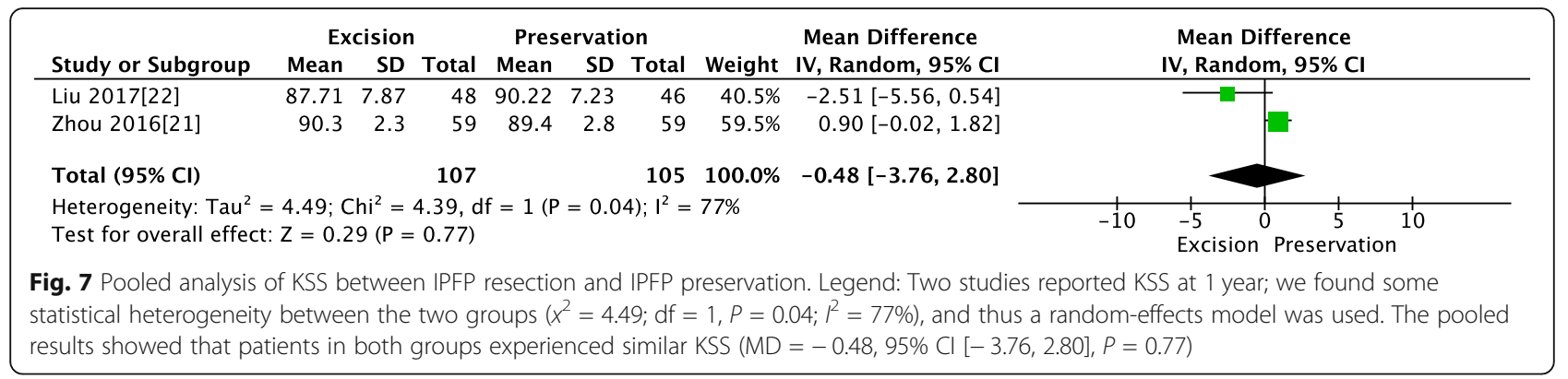

studies. There was no difference found for our study comparing post-operative KSS scores between patients undergoing resection and those with IPFP preservation which agreed with the previous metaanalysis

To the best of our knowledge, this is the first meta-analysis comparing outcomes between IPFP resection and IPFP preservation for TKA that only includes randomized controlled trials. However, it has the following limitations. First, the data used in this study was derived from several studies evaluating different surgical techniques by different surgeons. As such, the technique and surgeons, although similar, were not identical. Second, while we did not observe any publication bias, we acknowledge, as others have, that results should be treated with caution when meta-analyses are based on a limited number of small trials, as is the case in the current investigation. Third, conducting an actual RCT is difficult. Only nine studies were RCTs, and the blinding of participants and personnel in the studies was difficult. Some papers did not report all of our outcomes of interest.
There are several strengths of this study that warrant mention: (1) Compared with the previous meta-analysis, we included some new clinical research up to March 2020; our results are therefore more up to date. (2) All included studies are RCTs which directly compared IPFP $R$ and IPFP P. (3) Reporting was conducted on many new outcome measures such as range of motion and ISR which could be a supplement for the previous metaanalysis. (4) Some outcomes were sub-analyzed by categories of follow-up time.

\section{Conclusion}

Based on the available level I evidence, we are unable to conclude whether resection or preservation should be the better operative choice. So we advised that surgeons could keep the fat pad if excellent exposure can be achieved but resect it if needed to improve exposure during TKA. More evidence is needed before one surgical technique can be definitively considered superior. We required more adequately powered and betterdesigned RCT studies with long-term follow-up to reach a firmer conclusion.

\begin{tabular}{|c|c|c|c|c|c|c|c|c|c|}
\hline \multirow{3}{*}{$\begin{array}{l}\text { Study or Subgroup } \\
5.1 .16 \text { months }\end{array}$} & \multicolumn{3}{|c|}{ Excision } & \multicolumn{3}{|c|}{ Preservation } & \multirow{3}{*}{ Weight } & \multirow{3}{*}{$\begin{array}{l}\text { Mean Difference } \\
\text { IV, Random, } 95 \% \mathrm{CI}\end{array}$} & \multirow{2}{*}{$\begin{array}{c}\text { Mean Difference } \\
\text { IV, Random, } 95 \% \mathrm{Cl}\end{array}$} \\
\hline & Mean & SD & Total & Mean & SD & Total & & & \\
\hline & & & & & & & & & \\
\hline Li 2017[20] & 122 & 16 & 51 & 126 & 16 & 53 & $8.9 \%$ & $-4.00[-10.15,2.15]$ & \\
\hline $\begin{array}{l}\text { Tanaka } 2003[17] \\
\text { Subtotal }(\mathbf{9 5 \%} \mathrm{Cl})\end{array}$ & 123 & 2.75 & $\begin{array}{r}54 \\
105\end{array}$ & 123 & 3.25 & $\begin{array}{r}53 \\
106\end{array}$ & $\begin{array}{l}34.8 \% \\
43.7 \%\end{array}$ & $\begin{array}{r}0.00[-1.14,1.14] \\
-0.81[-3.96,2.34]\end{array}$ & \\
\hline \multicolumn{10}{|c|}{$\begin{array}{l}\text { Heterogeneity: } \mathrm{Tau}^{2}=2.91 ; \mathrm{Chi}^{2}=1.57, \mathrm{df}=1(\mathrm{P}=0.21) ; \mathrm{I}^{2}=36 \% \\
\text { Test for overall effect: } \mathrm{Z}=0.50(\mathrm{P}=0.61)\end{array}$} \\
\hline \multicolumn{10}{|l|}{ 5.1.2 1 year } \\
\hline Li 2017[18] & 116.21 & 0.51 & 38 & 113.92 & 0.25 & 38 & $38.7 \%$ & $2.29[2.11,2.47]$ & $\boldsymbol{\square}$ \\
\hline $\begin{array}{l}\text { Liu } 2017[22] \\
\text { Subtotal }(95 \% \mathrm{CI})\end{array}$ & 124.5 & 9.94 & $\begin{array}{l}48 \\
86\end{array}$ & 125.52 & 8.15 & $\begin{array}{l}46 \\
84\end{array}$ & $\begin{array}{l}\mathbf{1 7 . 7 \%} \\
\mathbf{5 6 . 3} \%\end{array}$ & $\begin{array}{r}-1.02[-4.69,2.65] \\
\mathbf{1 . 1 6}[-\mathbf{1 . 9 1}, 4.24]\end{array}$ & \\
\hline \multicolumn{10}{|c|}{$\begin{array}{l}\text { Heterogeneity: } \mathrm{Tau}^{2}=3.72 ; \mathrm{Chi}^{2}=3.12, \mathrm{df}=1(\mathrm{P}=0.08) ; \mathrm{I}^{2}=68 \% \\
\text { Test for overall effect: } \mathrm{Z}=0.74(\mathrm{P}=0.46)\end{array}$} \\
\hline Total $(95 \% \mathrm{Cl})$ & & & 191 & & & 190 & $100.0 \%$ & $0.35[-1.74,2.44]$ & \\
\hline \multicolumn{9}{|c|}{$\begin{array}{l}\text { Heterogeneity: } \mathrm{Tau}^{2}=2.93 ; \mathrm{Chi}^{2}=22.03, \mathrm{df}=3(\mathrm{P}<0.0001) ; \mathrm{I}^{2}=86 \% \\
\text { Test for overall effect: } \mathrm{Z}=0.33(\mathrm{P}=0.74) \\
\text { Test for subgroup differences: } \mathrm{Chi}^{2}=0.77, \mathrm{df}=1(\mathrm{P}=0.38), \mathrm{I}^{2}=0 \%\end{array}$} & $\begin{array}{ccc}-5 & 1 & 1 \\
\text { Excision } & \text { Preservation }\end{array}$ \\
\hline \multicolumn{10}{|c|}{$\begin{array}{l}\text { Fig. } 8 \text { Pooled analysis of knee range of movements between IPFP resection and IPFP preservation. Legend: Two studies reported a knee range of } \\
\text { movements within } 6 \text { months, and two studies reported a knee range of movements in } 1 \text { year. The pooled results showed that patients in both } \\
\text { groups experienced a similar knee range of movements within } 6 \text { months (MD }=-0.81,95 \% \mathrm{Cl}[-3.96,2.34], P=0.61) \text { and at } 1 \text { year }(\mathrm{MD}=1.16 \text {, } \\
95 \% \mathrm{Cl}[-1.91,4.24], P=0.46)\end{array}$} \\
\hline
\end{tabular}




\section{Abbreviations}

Cls: Confidence intervals; RCTs: Randomized controlled trials; TKA: Total knee arthroplasty; IPFP: Infrapatellar fat pad; PTL: Patellar tendon length; ISR: InsallSalvati ratio; AKP: Anterior knee pain; KSS: Knee Society Scores; ROM: Range of motion; PRISMA: Preferred Reporting Items for Systematic Reviews and Meta-analyses

\section{Acknowledgements}

Not applicable.

\section{Authors' contributions}

CJS, HDY, and XC were responsible for conception and design of the study and drafted the manuscript. CJS, YT, and HML contributed to data collection. CJS and HDY. contributed to language editing. CJS and XFZ contributed to manuscript preparation and data analysis. HML and HDY contributed to study retrieval. CJS, XFZ, and HDY contributed to revision of the manuscript. All authors read and approved the final manuscript.

\section{Funding}

None.

\section{Availability of data and materials}

The datasets generated and analyzed during the current study are available from the corresponding author on reasonable request.

\section{Ethics approval and consent to participate}

Not applicable.

\section{Consent for publication}

Not applicable.

\section{Competing interests}

The authors declare that they have no competing interests.

\section{Author details}

${ }^{1}$ Department of Orthopedic, Beijing Tsinghua Changgung Hospital, School of Clinical Medicine, Tsinghua University, No.168 Litang Road, Dongxiaokou Town, Changping District, Beijing 102218, China. ${ }^{2}$ Department of Clinical Epidemiology and Biostatistics, Beijing Tsinghua Changgung Hospital, School of Clinical Medicine, Tsinghua University, No.168 Litang Road, Dongxiaokou Town, Changping District, Beijing 102218, China. ${ }^{3}$ Department of Orthopedic, Kuching Specialist Hospital, Tabuan Stutong Commercial Centre, 93350 Kuching, Sarawak, Malaysia.

Received: 24 June 2020 Accepted: 29 July 2020

Published online: 05 August 2020

\section{References}

1. Lemon M, Packham I, Narang K, Craig DM. Patellar tendon length after knee arthroplasty with and without preservation of the infrapatellar fat pad. J Arthroplast. 2007;22:574-80. https://doi.org/10.1016/j.arth.2006.04.026.

2. Baker $\mathrm{PN}$, et al. The role of pain and function in determining patient satisfaction after total knee replacement. Data from the National Joint Registry for England and Wales. J Bone Joint Surg (Br). 2007;89:893-900. https://doi.org/10.1302/0301-620X.89B7.19091.

3. Dragoo $\mathrm{J}$, Johnson C, McConnell J. Evaluation and treatment of disorders of the infrapatellar fat pad. Sports Med. 2012;42:51-67. https://doi.org/10. 2165/11595680-000000000-00000

4. Davies GS, et al. Changes in patella tendon length over 5 years after different types of knee arthroplasty. Knee Surg Sports Traumatol Arthrosc. 2016;24:3029-35. https://doi.org/10.1007/s00167-016-4170-6.

5. McMahon, M. S. et al. Scintigraphic determination of patellar viability after excision of infrapatellar fat pad and/or lateral retinacular release in total knee arthroplasty. Clin Orthop Relat Res, 10-16 (1990).

6. Hughes SS, Cammarata A, Steinmann SP, Pellegrini VD Jr. Effect of standard total knee arthroplasty surgical dissection on human patellar blood flow in vivo: an investigation using laser Doppler flowmetry. J South Orthop Assoc. 1998:7:198-204.

7. Gandhi $R$, et al. Predictive risk factors for stiff knees in total knee arthroplasty. J Arthroplast. 2006:21:46-52. https://doi.org/10.1016/j.arth.2005. 06.004 .
8. Meneghini RM, et al. The effect of retropatellar fat pad excision on patellar tendon contracture and functional outcomes after total knee arthroplasty. J Arthroplast. 2007;22:47-50. https://doi.org/10.1016/j.arth.2007.03.031.

9. Pinsornsak P, Naratrikun K, Chumchuen S. The effect of infrapatellar fat pad excision on complications after minimally invasive TKA: a randomized controlled trial. Clin Orthop Relat Res. 2014;472:695-701. https://doi.org/10. 1007/s11999-013-3321-z.

10. Maculé F, et al. Hoffa's fat pad resection in total knee arthroplasty. Acta Orthop Belg. 2005;71:714-7.

11. Van Beeck A, et al. Does infrapatellar fat pad resection in total knee arthroplasty impair clinical outcome? A systematic review. Knee. 2013;20: 226-31. https://doi.org/10.1016/j.knee.2013.01.005.

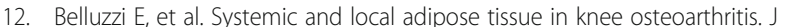
Cell Physiol. 2017;232:1971-8. https://doi.org/10.1002/jcp.25716.

13. White L, Holyoak R, Sant J, Hartnell N, Mullan J. The effect of infrapatellar fat pad resection on outcomes post-total knee arthroplasty: a systematic review. Arch Orthop Trauma Surg. 2016;136:701-8. https://doi.org/10.1007/ s00402-016-2440-x.

14. Ioan-Facsinay A, Kloppenburg M. An emerging player in knee osteoarthritis: the infrapatellar fat pad. Arthritis Res Ther. 2013;15:225. https://doi.org/10. 1186/ar4422.

15. Tanaka N, Sakahashi H, Sato E, Hirose K, Isima T. Influence of the infrapatellar fat pad resection in a synovectomy during total knee arthroplasty in patients with rheumatoid arthritis. J Arthroplast. 2003;18:897-902. https:// doi.org/10.1016/s0883-5403(03)00271-7.

16. Guogiang Li QF, Ge M, Duan Z, Chen X. Analysis of the effects of infrapatellar fat pad resection in total knee arthroplasty on curative effect and prognosis. J Clin Med. 2017:4:8963-6.

17. Bing Xin GF, Wang Z. The effect of retaining or removing the infrapatellar fat pad on the height of the patella after total knee arthroplasty. China Prac Med. 2016;3:46-7.

18. Guoqiang Li QF, Ge M, Duan Z, Chen X. The effect of infrapatellar fat pad preservation in total knee arthroplasty. Shanxi Med J. 2017:46:3047-50.

19. Peng Zhou HZ, Wu Y, Wu H, Chen Y, Ding Z. The effect of infrapatellar fat pad resection in total knee arthroplasty. Orthopaedics. 2016;7:303-7.

20. Yan Liu YZ, Liu A, Liu J, Wang L, Zhang D, Tian Z. The effect of preserving or resection of the infrapatellar fat pad on the early efficacy and patellar tendon length after total knee replacement: a randomized controlled trial. Chin J Orthop. 2017:37:611-9.

21. Nisar S, Lamb JN, Somashekar N, Pandit H, van Duren BH. Preservation vs. resection of the infrapatellar fat pad during total knee arthroplasty part II: a systematic review of published evidence. Knee. 2019;26:422-6. https://doi. org/10.1016/j.knee.2019.01.007

22. Paulos LE, Rosenberg TD, Drawbert J, Manning J, Abbott P. Infrapatellar contracture syndrome. An unrecognized cause of knee stiffness with patella entrapment and patella infera. Am J Sports Med. 1987;15:331-41. https:// doi.org/10.1177/036354658701500407

23. Blackburne JS, Peel TE. A new method of measuring patellar height. J Bone Joint Surg (Br). 1977;59:241-2.

24. Caton J, Deschamps G, Chambat $P$, Lerat JL, Dejour H. Patella infera Apropos of 128 cases. Rev Chir Orthop Reparatrice Appar Mot. 1982;68:31725.

25. Hozack WJ, Goll SR, Lotke PA, Rothman RH, Booth RE Jr. The treatment of patellar fractures after total knee arthroplasty. Clin Orthop Relat Res. 1988, 123-27.

\section{Publisher's Note}

Springer Nature remains neutral with regard to jurisdictional claims in published maps and institutional affiliations. 